\title{
Simulating phi-4 scalar field on quantum computers
}

Andy C. Y. Li (FNAL)

APS March Meeting 2021 V34.00015

18 March 2021
Alexandru Macridin (FNAL)

Stephen Mrenna (FNAL)

Panagiotis Spentzouris (FNAL) 


\section{Scalar field theory with $\phi^{4}$ interaction}

- Lagrangian density $\quad \mathcal{L}=\frac{1}{2}\left(\partial_{0} \phi\right)^{2}-\frac{1}{2}(\nabla \phi)^{2}-\frac{1}{2} m_{0}^{2} \phi^{2}-\frac{\lambda}{4 !} \phi^{4}$

- Discretized model in $(d+1)$-dimension with lattice spacing $a$

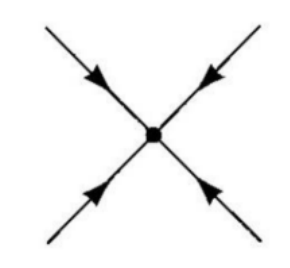

$$
H=\sum_{j}\left[\frac{1}{2} \Pi_{j}^{2}+\frac{1}{2}\left(m_{0 r}^{2}+2 d\right) \Phi_{j}^{2}-\sum_{e=1}^{d} \Phi_{j} \Phi_{j+e}+\frac{g_{0}}{4 !} \Phi_{j}^{4}\right] \quad m_{0 r}^{2} \equiv m_{0}^{2} a^{2} \quad \text { and } \quad g_{0} \equiv \lambda a^{3-d}
$$

- Continuous model with Lorentz invariance recovers in the limit $a \rightarrow 0$

- Outline:

- Binary encoding in position basis

- State preparation: vacuum state
Quantum Algorithms for Quantum Field Theories

Stephen P. Jordan ${ }^{1, *}$, Keith S. M. Lee ${ }^{2}$, John Preskill ${ }^{3}$

+ See all authors and affiliations

Science 01 Jun 2012:
Vol. 336 , Issue 6085, pp. $1130-1133$
DOl: $10.1126 /$ science. 1217069 


\section{Binary encoding in position basis}

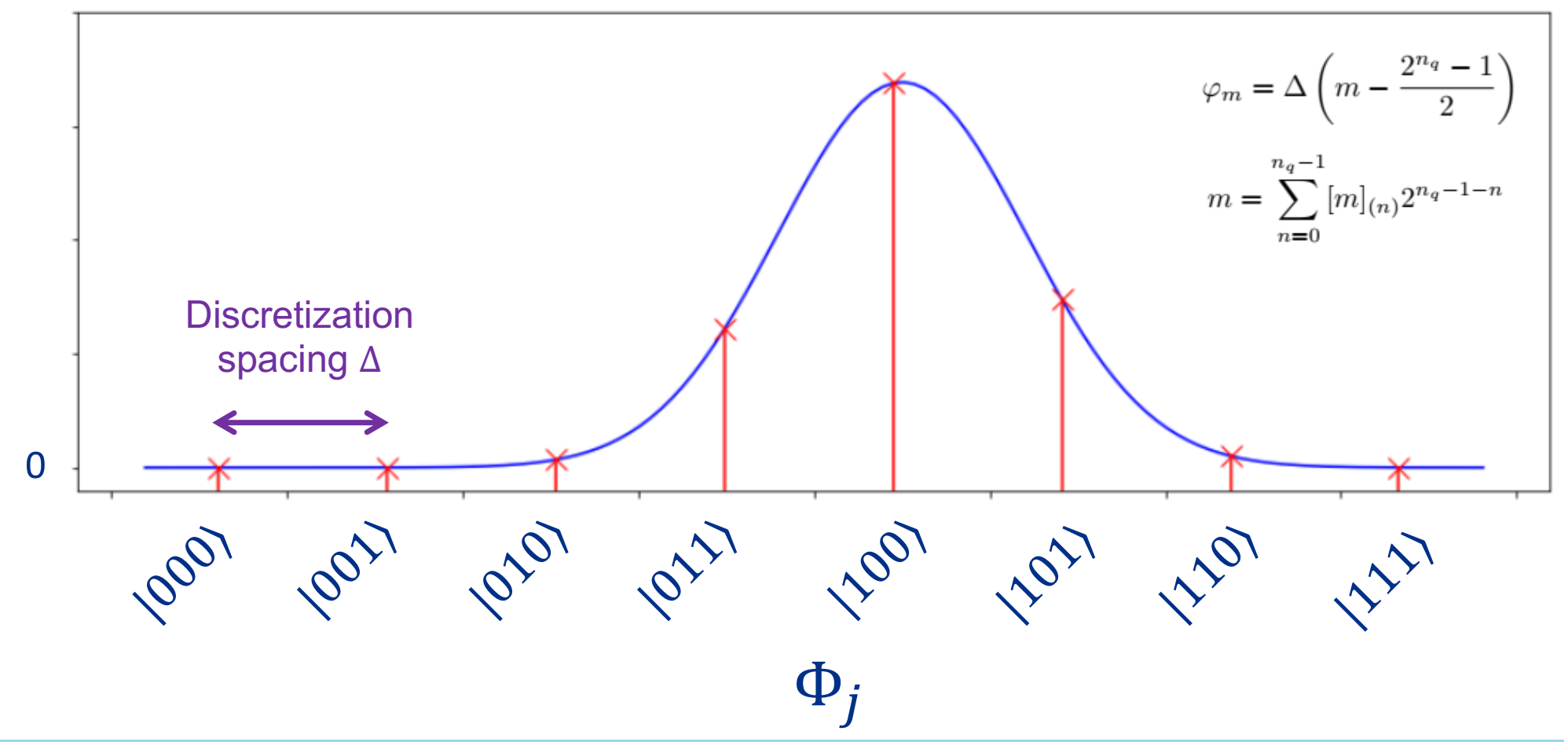




\section{Field operators in binary encoding}

- Discretization and binary representation

$$
\varphi_{m}=\Delta\left(m-\frac{2^{n_{q}}-1}{2}\right) \quad m=\sum_{n=0}^{n_{q}-1}[m]_{(n)^{n^{n_{q}-1-n}}}
$$

- Field operator $\Phi=\Delta\left(\sum_{n=0}^{n_{q}-1} \frac{-\sigma_{n}^{z}+1}{2} 2^{n_{q}-1-n}-\frac{2^{n_{q}}-1}{2}\right)=\Delta \sum_{n=0}^{n_{q}-1} \frac{-\sigma_{n}^{z}}{2} 2^{n_{q}-1-n}$

$$
e^{-i \theta \Phi}=e^{-i \sum_{n=0}^{n_{q}-1} \frac{-\sigma_{n}^{z}}{2} 2^{n_{q}-1-n} \Delta \theta}=\prod_{n=0}^{n_{q}-1} \mathrm{R}_{\mathrm{Z}}^{(n)}\left(-2^{n_{q}-1-n} \Delta \theta\right)
$$

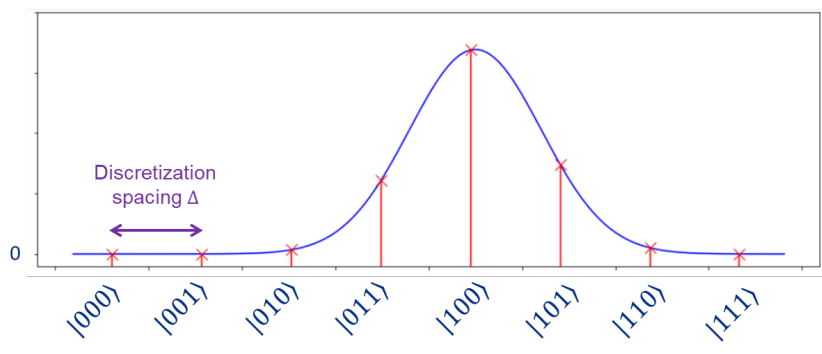

$\Phi_{j}$

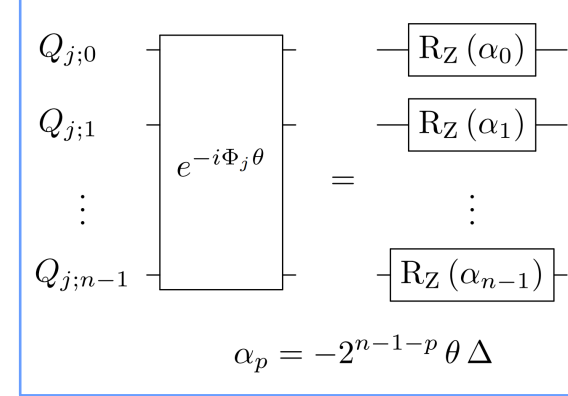

- Field operator square

$$
\begin{gathered}
\Phi^{2}=\Delta^{2}\left[\sum_{m<n} \frac{-\sigma_{m}^{z}+1}{2} \frac{-\sigma_{n}^{z}+1}{2} 2^{2 n_{q}-1-m-n}+\sum_{n=0}^{n_{q}-1} \frac{-\sigma_{n}^{z}}{2} 2^{n_{q}-1-n}\left(2^{n_{q}-1-n}-2^{n_{q}}+1\right)\right]+\phi_{2} \\
\text { Control-phase gates } \\
\text { Z-rotation gates }
\end{gathered}
$$

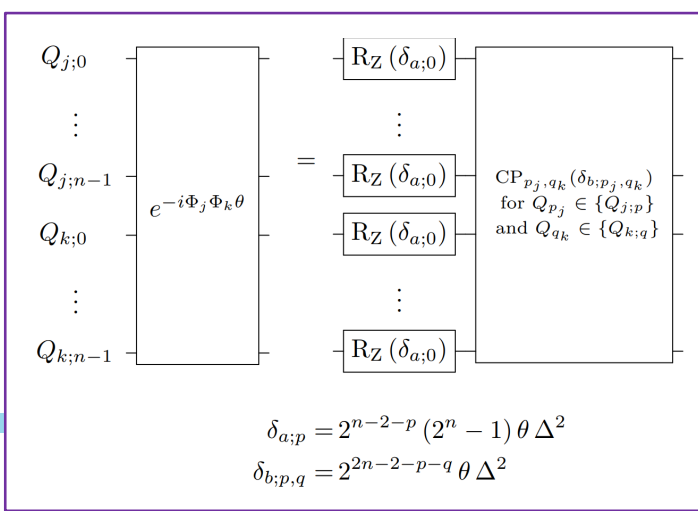




\section{Field operators: gate counts}

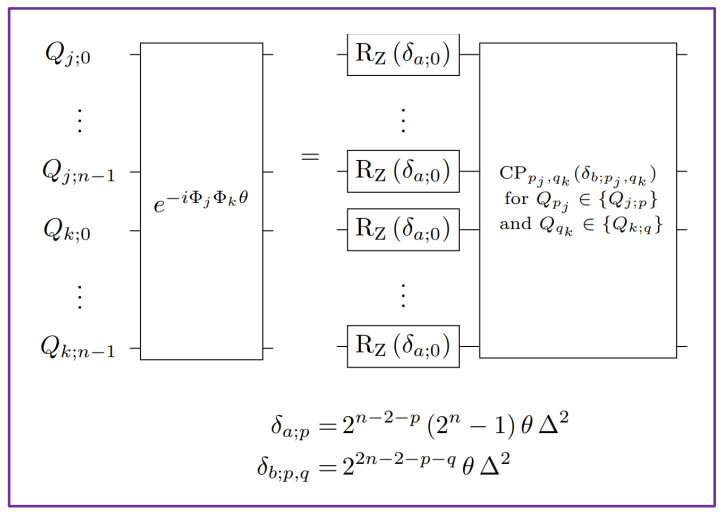

\begin{tabular}{|c|c|c|}
\hline & CPHASE & CNOT \\
\hline$e^{-i \Phi \theta}$ & 0 & 0 \\
\hline$e^{-i \Phi^{2} \theta}$ & $n(n-1) / 2$ & 0 \\
\hline$e^{-i \Phi^{4} \theta}$ & $n(n-1) / 2$ & $n\left(n^{3}-6 n^{2}+11 n-6\right) / 4$ \\
\hline$e^{-i \Phi_{j} \Phi_{k} \theta}$ & $n^{2}$ & 0 \\
\hline
\end{tabular}

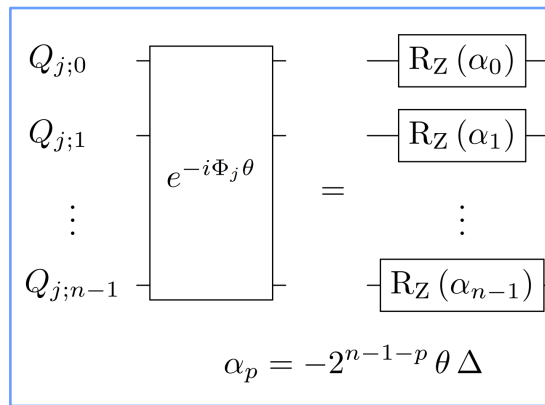

$$
H=\sum_{j}\left[\frac{1}{2} \Pi_{j}^{2}+\frac{1}{2}\left(m_{0 r}^{2}+2 d\right) \Phi_{j}^{2}-\sum_{e=1}^{d} \Phi_{j} \Phi_{j+e}+\frac{g_{0}}{4 !} \Phi_{j}^{4}+f \Phi_{j}\right]
$$

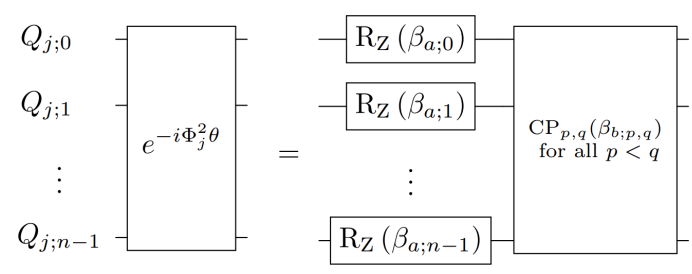

$\mathrm{CP}_{p, q}(\beta)=e^{i \beta|11 \chi 11|}=$ Control-Phase gate on $Q_{j ; p}$ and $Q_{j ; q}$ $\beta_{a ; p}=-2^{n-1-p}\left(2^{n-1-p}-2^{n}+1\right) \theta \Delta^{2}$

$\beta_{b ; p, q}=-2^{2 n-1-p-q} \theta \Delta^{2}$

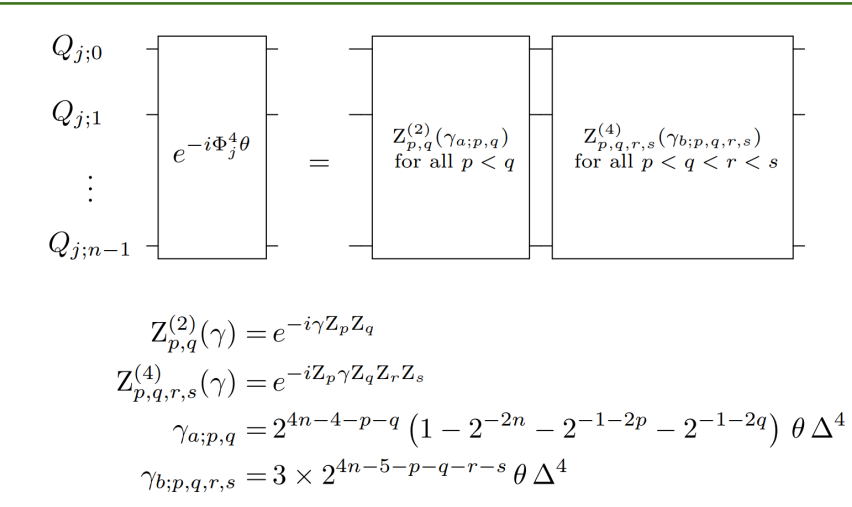

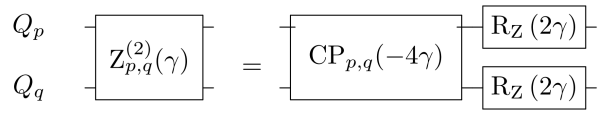

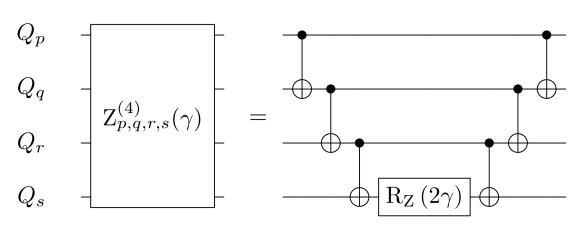




\section{Time evolution in binary encoding}

- $f\left(\Pi_{j}\right)=\mathrm{FT}^{-1} f\left(\Phi_{j}\right) \mathrm{FT}$

$$
H=\sum_{j}\left[\frac{1}{2} \Pi_{j}^{2}+\frac{1}{2}\left(m_{0 r}^{2}+2 d\right) \Phi_{j}^{2}-\sum_{e=1}^{d} \Phi_{j} \Phi_{j+e}+\frac{g_{0}}{4 !} \Phi_{j}^{4}+f \Phi_{j}\right]
$$

- $\mathrm{FT}=\prod_{p} R_{Z}\left(\pi \frac{n-1}{2 n} p\right) \quad \mathrm{QFT} \prod_{p} R_{Z}\left(\pi \frac{n-1}{2 n} p\right)$

- Cost of $\mathrm{QFT}=\frac{\mathrm{n}(\mathrm{n}-1)}{2}$ CPHASE gates

\begin{tabular}{|c|c|c|}
\hline & CPHASE & CNOT \\
\hline$e^{-i \Phi \theta}$ & 0 & 0 \\
\hline$e^{-i \Phi^{2} \theta}$ & $n(n-1) / 2$ & 0 \\
\hline$e^{-i \Phi^{4} \theta}$ & $n(n-1) / 2$ & $n\left(n^{3}-6 n^{2}+11 n-6\right) / 4$ \\
\hline
\end{tabular}

- 1 first-order Trotter step with 32 states per

\begin{tabular}{c|c|c}
\hline$e^{-i \Phi_{j} \Phi_{k} \theta}$ & $n^{2}$ & 0 \\
$e^{-i \Pi^{2} \theta}$ & $3 n(n-1) / 2$ & 0
\end{tabular}
75M CPHASE, 30M CNOT 


\section{Vacuum state preparation: local variational + adiabatic transfer}

$$
H=\sum_{j}\left[\frac{1}{2} \Pi_{j}^{2}+\frac{1}{2}\left(m_{0 r}^{2}+2 d\right) \Phi_{j}^{2}+\frac{g_{0}}{4 !} \Phi_{j}^{4}-\sum_{e=1}^{d} \Phi_{j} \Phi_{j+e}\right]
$$

Variational preparation of ground state

\section{Adiabatically switch on $H_{1}$}

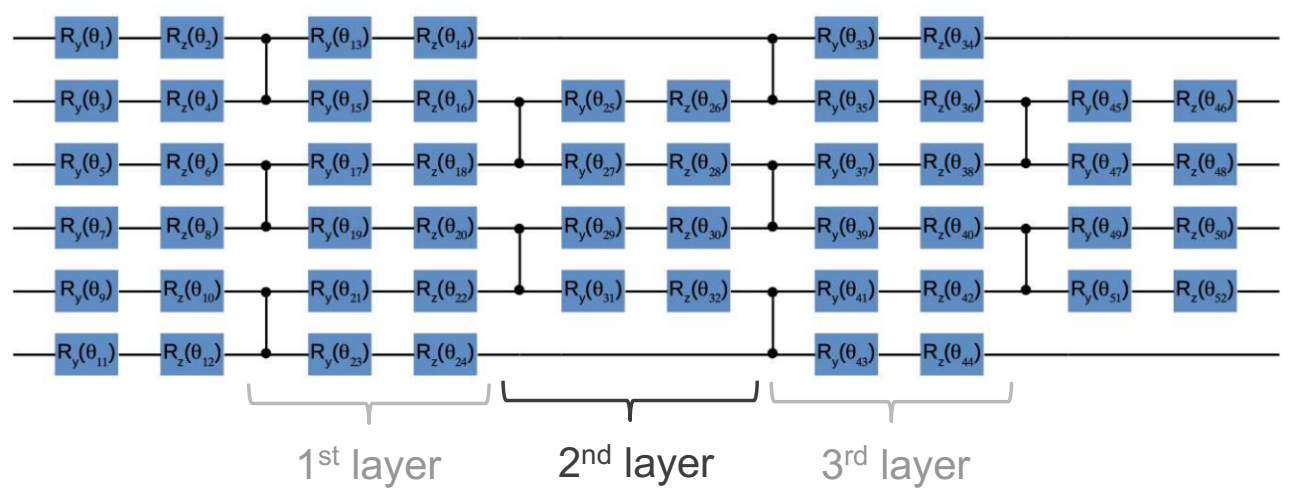

\section{Advantage:}

1. Efficient variational preparation compared to Kitaev-Webb method [arXiv:0801.0342 (2008)]

2. Prepare the vacuum state for any $\lambda_{0}$ and $m_{0}^{2}$ (including $m_{0}^{2}<0$ ) 


\section{High fidelity local state preparation by hardware efficient ansatz}
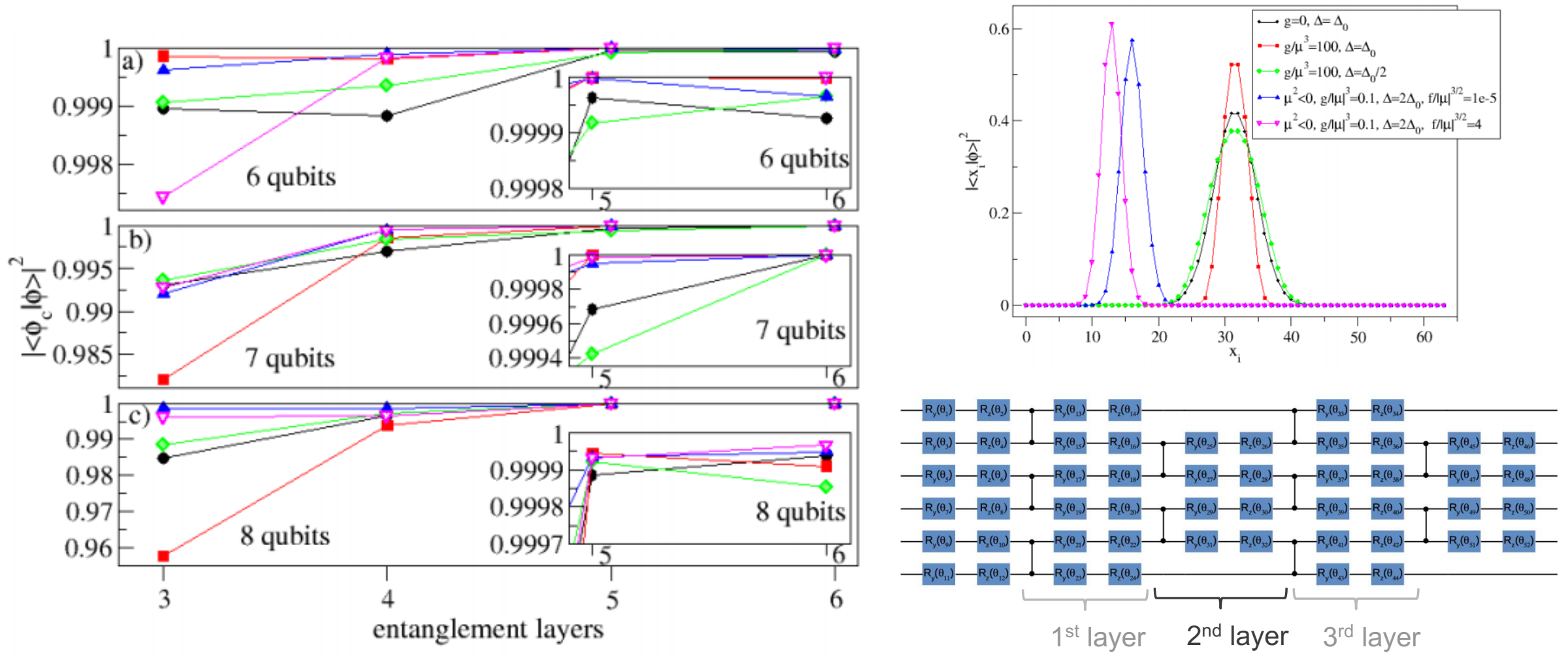


\section{Adiabatic state transfer in the broken-symmetry phase}

$H=\sum_{j}\left[\frac{1}{2} \Pi_{j}^{2}+\frac{1}{2}\left(m_{0 r}^{2}+2 d\right) \Phi_{j}^{2}+\frac{g_{0}}{4 !} \Phi_{j}^{4}-\sum_{e=1}^{d} \Phi_{j} \Phi_{j+e}\right]$

- Renormalization large $\lambda \rightarrow m_{0 r}^{2}<0$

- Broken symmetry phase $\left\langle\Phi_{j}\right\rangle \neq 0$ double-well potential degenerate ground states

Adiabatically switch off

$$
H=\sum_{j}\left[\frac{1}{2} \Pi_{j}^{2}+\frac{1}{2}\left(m_{0 r}^{2}+2 d\right) \Phi_{j}^{2}+\frac{g_{0}}{4 !} \Phi_{j}^{4}+f \Phi_{j}-\sum_{e=1}^{d} \Phi_{j} \Phi_{j+e}\right]
$$$$
\text { Adiabatically switch on }
$$

Variational state preparation
PHYSICAL REVIEW D

VOLUME 13 , NUMBER 10

Existence of a second-order phase transition in a two-dimensional $\phi^{4}$ field theory*

Shau-Jin Chang ${ }^{\dagger}$
Department of Physics, University of Illinois at Urbana-Champaign, Urbana, Illinois 61801 (Received 9 February 1976)

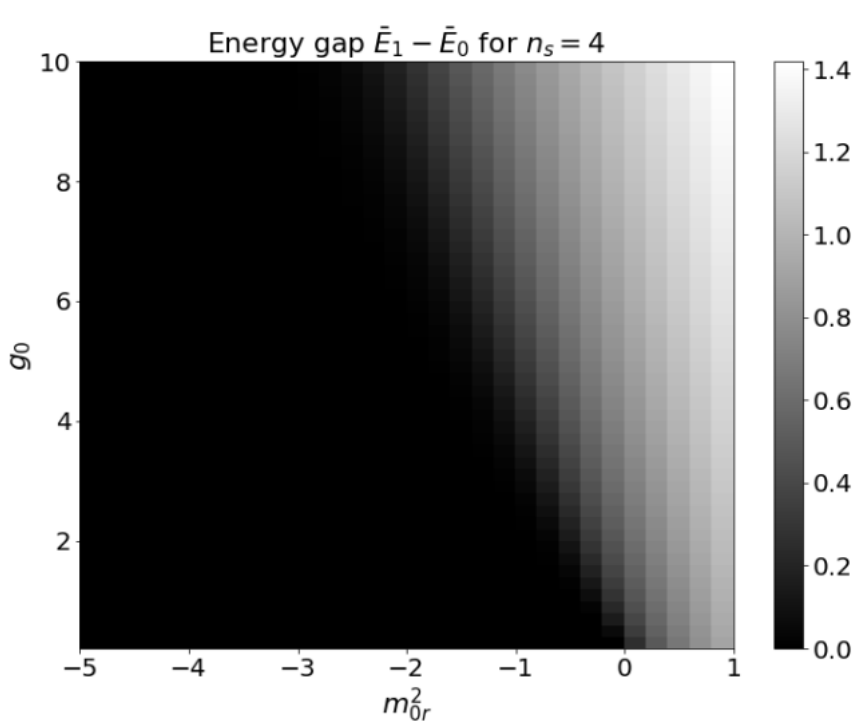




\section{Adiabatic time required to prepare broken-symmetry states}
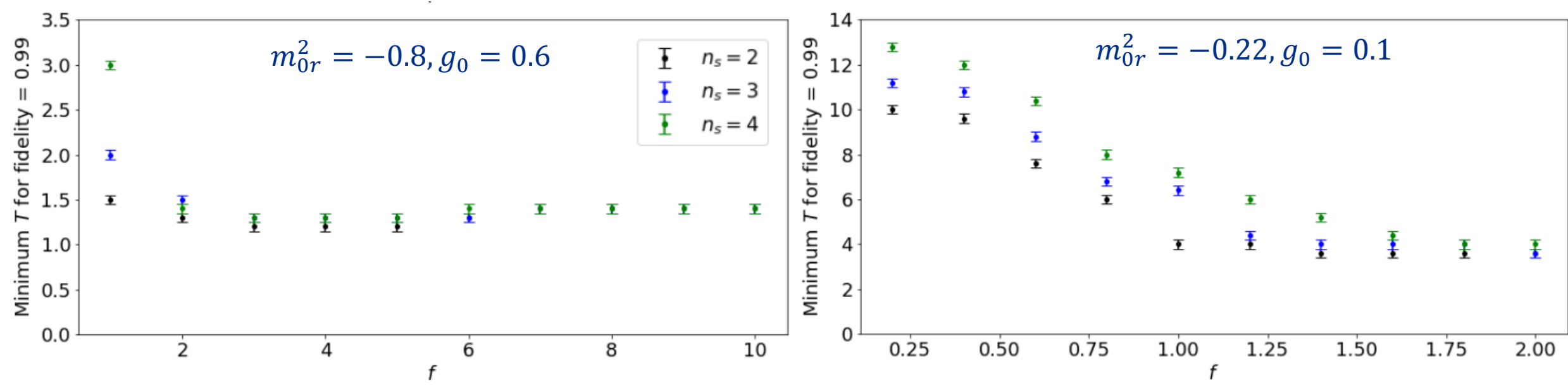

- Continuous limit: $a \rightarrow 0, g_{0}=\lambda a^{3-d} \rightarrow 0$ for 1D and 2D systems

- State preparation becomes more difficult due to stronger effective site-site coupling

- System size dependence: require further study

- Appears to be less sensitive with a sufficiently large $f$ 


\section{Summary}

- Quantum simulation of $\phi^{4}$ scaler field theory using binary encoding in the position basis

- Variational hardware-efficient ansatz: flexible tool to prepare the local ground state with very high fidelity

- Flexibility: design local Hamiltonian to further optimize the algorithm

- Adiabatic state transfer: connect the engineered local Hamiltonian to the full Hamiltonian

- Gate count: require better QPU to be implemeneted

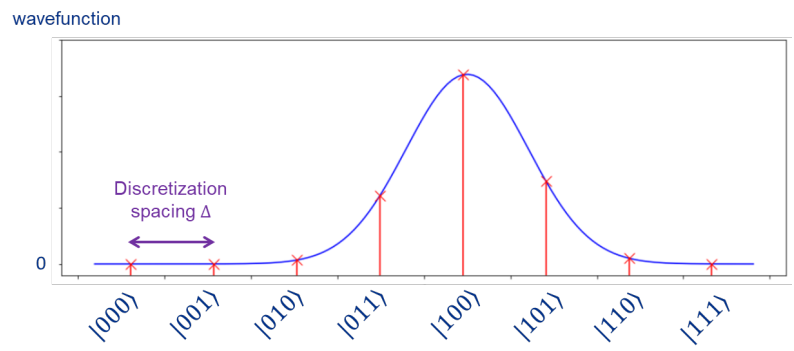

$\Phi_{j}$
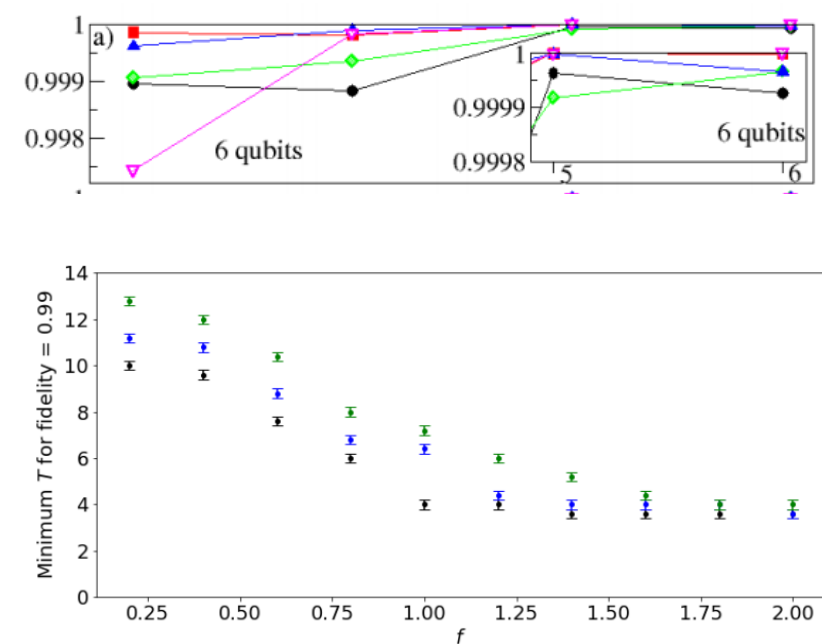